\title{
Local density of states for the corner geometry interface of $d$-wave superconductors, within the extended Hubbard model
}

\author{
N. Stefanakis \\ Department of Physics, University of Crete, P.O. Box 2208, GR-71003, Heraklion, Crete, Greece
}

(October 29, 2018)

\begin{abstract}
The spatial variations of the order parameter, and the local density of states (LDOS) on the corner of $s$-wave or $d_{x^{2}-y^{2}}$-wave superconductors, as well as in superconductor-insulator-normal metal interfaces, are calculated self consistently using the Bogoliubov-deGennes formalism within the two dimensional extended Hubbard model. The exact diagonalization method is used. Due to the suppression of the dominant $d$-wave order parameter, the extended $s$ wave order parameter is induced near the surface, that alternates its sign for the topmost sites at adjacent edges of the lattice and decays to zero in the bulk. The presence of surface roughness results into the appearance of the zero band conduction peak (ZBCP) near the corner surface which lacks from the predictions of the quasiclassical theory.
\end{abstract}

Typeset using REVTEX 


\section{INTRODUCTION}

The determination of the order parameter symmetry has become one of the main aspects in the research on high temperature superconductors [1],2]. Tunneling conductance experiments report the existence of a zero band conduction peak (ZBCP) [3,4]. The origin of the experimental ZBCP is explained in the context of zero energy states (ZES) [5] formed near the [110] surfaces of $d$-wave superconductors [6.77. These ZES do not appear for $s$-wave superconductors or near the [100] surface of $d$-wave superconductors and are one of the features that characterize the $d$-wave superconductors.

The quasiclassical theory of superconductivity has been used to calculate the tunneling conductance in interfaces of unconventional superconductors with normal metals or ferromagnets 8-10]. In the quasiclassical approximation the quasiparticles move in classical trajectories (external degrees of freedom) with internal degrees of freedom which are the spin and the particle-hole degrees of freedom. The orientation dependence of the spectra as well as the $V$ line shape of the conductance curve are explained by the formation of bound states close to the interface due to the sign change of the pair potential that the transmitted quasiparticles experience.

Moreover the concept of phase shift by $\pi$ of the order parameter in orthogonal directions in $k$-space, which is equivalent to the sign change of the Josephson critical current, can be observed in corner junctions of anisotropic superconductors with conventional s-wave superconductors as a dip of the Fraunhofer pattern at zero magnetic field [0]. It is an indication of $d$-wave symmetry of the order parameter. The spontaneous flux modulation with surface orientation in such junctions has been calculated and can be used to distinguish the subdominant components $s$ or $d_{x y}$ that are induced at regions where the $d$-wave order parameter is suppressed.

The extended Hubbard model has been used to study single vortex structure [11,12], time reversal symmetry breaking across twin boundaries [13] or near surfaces [7], the effect of disorder [14], and the effect of surface roughness [6]. In this paper the Bogoliubov-deGennes 
equations are solved in a two dimensional square lattice within the context of an extended Hubbard model. The spatial variation of order parameter, and the local density of states, which in the limit of low transparency barrier converges to the tunneling conductance, are calculated for various types of surfaces and interfaces e.g. a corner surface, the interface of $d$ wave or $s$-wave superconductor along the [110] direction with normal metals. The evolution of the local density of states (LDOS) is studied as a function of the distance from the surface.

It is seen that the extended $s$-wave order parameter is induced due to the suppression of the dominant $d$-wave order parameter which alternates its sign for the topmost sites at adjacent edges of the lattice and decays to zero in the bulk. The LDOS is symmetric when $\mu=0$ and it becomes asymmetric when $\mu$ deviates from zero due to the breakdown of the electron-hole symmetry. We also investigate the effect of the surface roughness near the corner. In general surface roughness which in real samples is of atomic length scale modifies the properties of the quasiparticles since the coherence length of the cuprates is much smaller than the conventional $s$-wave superconductors. Our model treats the quasiparticle properties on atomic length scale and goes beyond the quasiclassical approximation. The presence of surface roughness results into the appearance of the ZBCP near the corner surface which lacks from the predictions of the quasiclassical theory.

The rest of the paper is organized as following. In Sec. II we develop the model and discuss the formalism. In Sec. III we present the results for the corner of superconductor. In Sec. IV we present the $s-I-n, d-I-n$ interfaces. In Sec. V, the effect of the surface roughness is considered. Finally, summary and discussions are presented in the last section.

\section{BDG EQUATIONS, WITHIN THE HUBBARD MODEL}

The Hamiltonian for the extended Hubbard model on a two dimensional square lattice is

$$
H=-t \sum_{<i, j>\sigma} c_{i \sigma}^{\dagger} c_{j \sigma}+\mu \sum_{i \sigma} n_{i \sigma}+\sum_{i \sigma} \mu_{i}^{I} n_{i \sigma}
$$




$$
+V_{0} \sum_{i} n_{i \uparrow} n_{i \downarrow}+\frac{V_{1}}{2} \sum_{<i j>\sigma \sigma^{\prime}} n_{i \sigma} n_{j \sigma^{\prime}},
$$

where $i, j$ are sites indices and the angle brackets indicate that the hopping is only to nearest neighbors, $n_{i \sigma}=c_{i \sigma}^{\dagger} c_{i \sigma}$ is the electron number operator in site $i, \mu$ is the chemical potential. $V_{0}, V_{1}$ are on site and nearest-neighbor interaction strength. Negative values of $V_{0}$ and $V_{1}$ mean attractive interaction and positive values mean repulsive interaction. When $V_{1}<0$ the pairing interaction gives rise to the $d$-wave superconductivity in a restricted parameter regime [12]. To simulate the effect of the depletion of the carrier density at the surface or impurities the site-dependent impurity potential $\mu^{I}\left(r_{i}\right)$ is set to a sufficiently large value at the surface sites. This prohibit the electron tunneling over these sites. Within the mean field approximation Eq. (四) is reduced to the Bogoliubov deGennes equations [15]:

$$
\left(\begin{array}{cc}
\hat{\xi} & \hat{\Delta} \\
\hat{\Delta}^{*} & -\hat{\xi}
\end{array}\right)\left(\begin{array}{c}
u_{n}\left(r_{i}\right) \\
v_{n}\left(r_{i}\right)
\end{array}\right)=\epsilon_{n}\left(\begin{array}{c}
u_{n}\left(r_{i}\right) \\
v_{n}\left(r_{i}\right)
\end{array}\right),
$$

with

$$
\begin{gathered}
\hat{\xi} u_{n}\left(r_{i}\right)=-t \sum_{\hat{\delta}} u_{n}\left(r_{i}+\hat{\delta}\right)+\left(\mu^{I}\left(r_{i}\right)+\mu\right) u_{n}\left(r_{i}\right), \\
\hat{\Delta} u_{n}\left(r_{i}\right)=\Delta_{0}\left(r_{i}\right) u_{n}\left(r_{i}\right)+\sum_{\hat{\delta}} \Delta_{\delta}\left(r_{i}\right) u_{n}\left(r_{i}+\hat{\delta}\right),
\end{gathered}
$$

where the gap functions are defined by

$$
\begin{gathered}
\Delta_{0}\left(r_{i}\right) \equiv V_{0}<c_{\uparrow}\left(r_{i}\right) c_{\downarrow}\left(r_{i}\right)>, \\
\Delta_{\delta}\left(r_{i}\right) \equiv V_{1}<c_{\uparrow}\left(r_{i}+\hat{\delta}\right) c_{\downarrow}\left(r_{i}\right)>,
\end{gathered}
$$

where $\hat{\delta}=\hat{x},-\hat{x}, \hat{y},-\hat{y}$. Equations (2) are subject to the self consistency requirements

$$
\begin{aligned}
\Delta_{0}\left(r_{i}\right) & =V_{0} \sum_{n} u_{n}\left(r_{i}\right) v_{n}^{*}\left(r_{i}\right) \tanh \left(\frac{\beta \epsilon_{n}}{2}\right), \\
\Delta_{\delta}\left(r_{i}\right) & =\frac{V_{1}}{2} \sum_{n}\left(u_{n}\left(r_{i}+\hat{\delta}\right) v_{n}^{*}\left(r_{i}\right)\right. \\
& \left.+u_{n}\left(r_{i}\right) v_{n}^{*}\left(r_{i}+\hat{\delta}\right)\right) \tanh \left(\frac{\beta \epsilon_{n}}{2}\right) .
\end{aligned}
$$


We start with the approximate initial conditions for the gap functions (78,8). After exact diagonalization of Eq. (2) we obtain the $u\left(r_{i}\right)$ and $v\left(r_{i}\right)$ and the eigenenergies $\epsilon_{n}$. The quasiparticle amplitudes are then inserted into Eq. (7),8) and new gap functions $\Delta_{0}\left(r_{i}\right)$ and $\Delta_{\delta}\left(r_{i}\right)$ are evaluated. We reinsert these quantities into Eq. (3, (1) , and we proceed in the same way until we achieve selfconsistency, i.e., when the norm of the difference of $\Delta_{0}\left(r_{i}\right)$ and $\Delta_{\delta}\left(r_{i}\right)$ from their previous values is less than the desired accuracy. We then compute the $d$-wave and the extended $s$-wave gap functions given by the expressions [11]:

$$
\begin{aligned}
\Delta_{d}\left(r_{i}\right) & =\frac{1}{4}\left[\Delta_{\hat{x}}\left(r_{i}\right)+\Delta_{-\hat{x}}\left(r_{i}\right)-\Delta_{\hat{y}}\left(r_{i}\right)-\Delta_{-\hat{y}}\left(r_{i}\right)\right], \\
\Delta_{s}^{e x t}\left(r_{i}\right) & =\frac{1}{4}\left[\Delta_{\hat{x}}\left(r_{i}\right)+\Delta_{-\hat{x}}\left(r_{i}\right)+\Delta_{\hat{y}}\left(r_{i}\right)+\Delta_{-\hat{y}}\left(r_{i}\right)\right] .
\end{aligned}
$$

The number density at the $i$ th site is given by

$$
n_{i}=n_{i \uparrow}+n_{i \downarrow}=\sum_{n}\left[\left|u_{n}\left(r_{i}\right)\right|^{2} f\left(\epsilon_{n}\right)+\left|v_{n}\left(r_{i}\right)\right|^{2}\left(1-f\left(\epsilon_{n}\right)\right)\right],
$$

and the local density of states (LDOS) at the $i$ th site is given by

$$
\rho_{i}(E)=-2 \sum_{n}\left[\left|u_{n}\left(r_{i}\right)\right|^{2} f^{\prime}\left(E-\epsilon_{n}\right)+\left|v_{n}\left(r_{i}\right)\right|^{2} f^{\prime}\left(E+\epsilon_{n}\right)\right],
$$

where the factor 2 comes from the twofold spin degeneracy, $f^{\prime}$ is the derivative of the Fermi function,

$$
f(\epsilon)=\frac{1}{\exp \left(\epsilon / k_{B} T\right)+1}
$$

\section{CORNER OF SUPERCONDUCTOR}

In this section we study the spatial variation of the order parameter close to the corner surface of a two dimensional square lattice seen in Fig. [1. The different symmetries are introduced by varying the strength of the local and non-local pairing interaction constants $V_{0}, V_{1}$. 
We consider a two dimensional system of $30 \times 30$ sites, and we suppose fixed boundary conditions by setting the impurity potential $\mu^{I}=100 t$ at the surface. The temperature is $k_{B} T=0.1 t$. The constants $V_{0}$ and $V_{1}$ are 0.0 and $-2.5 t$ respectively. This choice of parameters gives $d$-wave superconductivity. The $\Delta_{d}$ order parameter is shown in Fig. 2, for two different values of the chemical potential $\mu=0$ and $\mu=t$. For $\mu=0$ the bulk value of the gap is $\Delta_{d}=0.32 t$, while the transition temperature is calculated as $k_{B} T_{c}=0.6 t$. It is much more suppressed for finite values of the chemical potential. As we can see the $d$-wave order parameter is enhanced near the surface from its bulk value, and goes to zero at the surface atoms because the hopping to these sites is difficult due to the impurity barrier. In Fig. 3 we see the induced extended $s$-wave order parameter $\Delta_{s}^{e x t}$, for two different values of the chemical potential $\mu=0$ and $\mu=t$. It is much more enhanced for greater values of the chemical potential. Near the surface $\Delta_{s}^{e x t}$ oscillates at an atomic scale and vanishes into the bulk region at a distance of few lattice sites. It reverses its sign on either side of the lattice edge and it is exactly zero in the diagonal direction. Next to the corner we see an enhancement from the edge value. It appears to have a $d$-wave like structure just at the corner of the square lattice. This behavior is also seen near impurities [14], and across twin boundaries [13] using BdG equations within the extended Hubbard model in a two dimensional orthorhombic lattice. The explanation beyond this is the sign change of the $d$-wave order parameter across the [110] direction close to the corner. The quasiparticles feel the sign change of the pairing potential when they are reflected from the lattice edges near the corner. In Fig. $1(\mathrm{a})$ we plot the local density of states for $\mu=0$ at points $A, B, C, D$ seen in Fig. [1, along the diagonal of the lattice. The symmetry with respect to $E=0$ is due to the zero chemical potential. The LDOS is site depended and shows a complicated gap structure. Also no ZBCP has been observed in agreement with the results of the quasiclassical theory 8 . As we move to the interior of the lattice the LDOS converges to the bulk density of states in a two dimensional square lattice. When $\mu$ deviates from zero as in Fig. $4(\mathrm{~b})$ the LDOS becomes asymmetric. However no ZBCP is formed. In Fig. 5(a) we plot the local density of states for $\mu=0$, at sites $x=1,2,3$ seen in Fig. 1, along the 
direction [100] perpendicular to the lattice edge. The LDOS has the $V$-like line shape, is symmetric with respect to $E=0$ due to the electron-hole symmetry, and has the minimum at $E=0$ which characterizes the $d$-wave symmetry of the order parameter. It is seen that the LDOS recovers its bulk value (long dashed line) as we move away from the surface. The symmetric form of the LDOS line shape is lost when the chemical potential deviates from zero, as seen in Fig. 5(b) for $\mu=t$.

To understand the effect of the different symmetry the local pairing interaction is set to the value $V_{0}=-2.5 t$, while the non-local interaction is set to the value $V_{1}=0$. This set of parameters gives rise to $s$-wave pairing. The spatial variation of the $s$-wave order parameter close to the corner of a two dimensional square lattice (see Fig. 6) is seen in Fig. 7, for two different values of the chemical potential $\mu=0$ and $\mu=t$. For $\mu=0$ as seen in Fig. W(a) the order parameter increases monotonically (which is to be compared to the $d$-wave case where this increase is non monotonous) to the bulk value at the sides of the lattice while at the corner an enhancement, relative to the bulk value is seen which disappears over a few sites to the interior of the lattice. For $\mu=t$ the order parameter increases non-monotonically even at the sides of the lattice as seen in Fig. 7(b). In Fig. Ba (b) we plot the local density of states at points $A, B, C, D$, for $\mu=0(\mu=t)$ seen in Fig. 6, along the diagonal direction of the lattice. The LDOS shows gap structure with $U$-like line shape which characterizes the $s$-wave symmetry and is symmetric (asymmetric) when $\mu$ is (deviates from) zero . The LDOS is insensitive to the orientation of the surface and is site independent. Similar behavior is seen for the [100] surface seen in Fig. 9.

The absence of ZBCP in the LDOS can be explained from the results of the quasiclassical theory as follows. The condition for the formation of $\mathrm{ZBCP}$ is the change of sign of the quasiparticles during the scattering from the surface of the superconductor. In $s$-wave superconductors this sign change does not occur at surfaces or interfaces due to the isotropy of the pair potential. In anisotropic superconductors this sign change is possible for certain orientation of the surface. However for the corner surface, at the direction where the lobes of the $d$-wave order parameter are at right angles to the surface, a typical trajectory of a 
quasiparticle would consist of two subsequent reflections from the lattices edges, in non of which sign change of the order parameter occurs. Therefore the quasiparticle does not feel the sign change of the order parameter and no ZBCP is formed. For a corner where the lobes are not exactly at right angles to the surface the condition for the formation of Andreev bound states at the surface can occur and also the ZBCP.

\section{IV. $S-I-N, D-I-N$ INTERFACES ALONG THE [110] DIRECTION}

We now discuss the effect of the interface on the order parameter and the local density of states for different symmetries. The interface is modeled by a line of sites along the diagonal of the lattice, $y^{\prime}$ direction, where the chemical potential is set to a value in accordance with the strength of the barrier we want to model. The value of the interaction strength in each part of the interface determines the particular system that we are considering.

To understand the effect of the symmetry of the pair potential we consider first the conventional $s$-wave superconductor- insulator-normal metal $(s-I-n)$ interface shown in Fig. 10(a). The local interaction in region where $x^{\prime}<0,\left(x^{\prime}\right.$ is the direction perpendicular to the interface) is $V_{0}=-2.5 t$, and the strength of the barrier is $\mu^{110}=100 t$. For $\mu=0$ the superconducting gap is $\Delta_{s}=0.6 t$. We plot in Fig. 10(b) the magnitude of the $s$-wave component $\Delta_{s}$ of the superconducting order parameter as a function of $x$ shown in Fig. 10(a) for two different values of the chemical potential, i.e., $\mu=0$ and $\mu=t$. It is suppressed near the interface and increases nonmonotonically to the bulk value at a few lattice sites. The enhancement at the topmost sites close to the interface is similar to the spatial variation of the $d$-wave superconductor close to the [100] surface seen in Fig. 2. The bulk order parameter is suppressed when $\mu$ deviates from 0 and also the spatial oscillations close to the interface are of reduced amplitude as seen in Fig. 10(b). We also plot in Fig. 10(c) the number density $n_{i}$ for the electrons for the two different values of the chemical potential, i.e., $\mu=0$ and $\mu=t$. For $\mu=0$ the number density is unity (one electron per site) in the bulk and decays to zero at the interface. However for finite $\mu$ the number density is reduced. 
In Fig. 11(b) we plot the local density of states (LDOS) at sites $A, B, C, D$, shown in Fig. 11(a), for $\mu=0$. As expected the LDOS line shape is symmetric since $\mu=0$. The LDOS shows the gap structure and $U$-like line shape. Also the LDOS is modified by the presence of the barrier, from it's bulk form. The LDOS is insensitive to the direction of the interface. For finite $\mu=1$ seen in Fig. 11](c) the LDOS keeps its $U$-like line shape. However it becomes asymmetric due to the breakdown of the electron-hole symmetry.

We consider then the unconventional $d$-wave superconductor-insulator-normal metal ( $d-$ $I-n)$ interface shown in Fig. 12(a) where $V_{1}=-2.5 t$, and $\mu^{110}=100 t$. We plot in Fig. 12(b) the magnitude of the $d$-wave component $\Delta_{d}$ and the magnitude of the extended $s$-wave component $\Delta_{s}^{e x t}$ of the order parameter as a function of the direction $x$ that is shown in Fig. 12(a), for two different values of the chemical potential $\mu=0$ and $\mu=t$. It is seen that for $\mu=0, \Delta_{s}^{e x t}$ is not modified by the presence of the interface. In contrast $\Delta_{d}$ drops to zero at the interface, and increases monotonically into a few lattice sites to the bulk value. For $\mu=t$ the $\Delta_{d}$ is much more suppressed close to the interface while the induced $\Delta_{s}^{e x t}$-wave component is more enhanced. Due to symmetry arguments the relation $\Delta_{\hat{x}}\left(r_{i}\right)=\Delta_{\hat{y}}\left(r_{i}\right)$ holds for the pairing potentials and results into a monotonic variation in the $d$-wave order parameter near the surface. This is different to the $s-I-n$ case, and also to the [100] surface of the $d$-wave superconductor seen in Fig. 2 where this increase is non-monotonous. In the latter case due to the translational invariance in the $y$-direction $\Delta_{-\hat{y}}\left(r_{i}\right)=\Delta_{\hat{y}}\left(r_{i}\right)$ is satisfied. The spatial oscillations are due to the atomic scale oscillation of the $\Delta_{\hat{x}}\left(r_{i}\right), \Delta_{-\hat{x}}\left(r_{i}\right)$ that are completely neglected in the quasiclassical approximation. We also plot in Fig. 12(c) the number density $n_{i}$ for the electrons for the two different values of the chemical potential, i.e., $\mu=0$ and $\mu=t$. It is similar to the case of the $s-I-n$-interface seen in Fig. 10(c)

The main difference between the two symmetries appear in the LDOS as we can see in Fig. 13(b) for the sites $A, B, C, D$, shown in Fig. 13(a), for $\mu=0$. As expected the LDOS line shape is symmetric since $\mu=0$. The ZBCP is formed and its height decreases exponentially as we move to the interior of the lattice along the direction perpendicular to the interface. However at site D no ZBCP is formed. The disappearance of ZBCP at 
D denotes that the zero energy states (ZES) wave functions have spatial variation close to the interface with nodes at specific sites. In this case site D corresponds to a node and therefore the ZBCP disappears. The ZBCP is explained in the context of zero energy states [5] formed near the [110] surfaces of $d$-wave superconductors due to the sign change that the quasiparticles experience in different directions in $k$ space. However the absence of ZBCP for

the [110] at specific sites is not predicted by the quasiclassical theory. For finite $\mu=1$ seen in Fig. 13(c) the LDOS keeps its $V$-like line shape. However it becomes asymmetric due to the breakdown of the electron-hole symmetry. Also the ZBCP is reduced. We conclude that the order parameter as well as the local density of states are influenced by the orientation of the interface [16].

\section{RESULT FOR THE SURFACE ROUGHNESS}

In the following we describe the effect of the surface roughness near the corner of the lattice. For the case of one step structure, for $\mu=0$, shown in Fig. [14(a) the LDOS shows ZBCP at points A,B but not in $\mathrm{C}$ as presented in Fig. 14(b). Moreover the ZBCP is suppressed compared to the case of flat [110] surface seen in Fig. 13. The quasiclassical theory predicts that for that direction the ZBCP is maximum [8] since the one step structure corresponds to the $a=\pi / 4$ (where $a$ is the orientation of the surface). The suppression of the ZBCP and also the disappearance of ZBCP from specific sites is explained by the spatial variation of the ZES. It is seen that the wave functions of the ZES form standing waves that decay in the bulk. The sites A and B, that show ZBCP, correspond to an antinode while for the rest of the lattice sites the amplitude of the ZES is zero. For finite $\mu$ the ZBCP disappears from sites A and B due to the destructive interference of the ZES as shown in Fig. 14(c). In addition the overall line shape of the conductance curve is asymmetric due to the breaking of the electron-hole symmetry. In Fig. 15 the spatial variation of the $d$ wave and extended $s$-wave order parameter is plotted for $\mu=0$ at sites close to the lattice corner. It is seen that the $d$-wave order parameter is suppressed at the impurity site while 
the extended $s$-wave order parameter is not much influenced.

For the $1 \times 2$ step structure shown in Fig. 16(a) the LDOS, presented in Fig. 16(b) for $\mu=0$, at points $\mathrm{A}, \mathrm{B}, \mathrm{C}, \mathrm{D}$ shows no ZBCP. The quasiclassical theory predicts a ZBCP since this geometry corresponds to a surface tilted from $a=0$ or $a=\pi / 2$. The absence of ZBCP is explained by the destructive interference of the standing waves and also by the asymmetricity of the structure. For $\mu=t$ shown in Fig. 16(c) some tiny conductance peak recovers.

The ZBCP at the topmost sites recovers for the geometry shown in Fig. 17(a) for sites A,C, for $\mu=0$ as presented in Fig. 17(b). However unlike the flat [110] surface no ZBCP is formed in site B due to the destructive interference of the standing waves. Moreover when finite chemical potential is introduced as shown in Fig. 17(c) the spatial distribution of the ZES is disturbed and for example the ZBCP appears even for sites, e.g., D, where normally for $\mu=0$ is absent.

\section{CONCLUSIONS}

We calculated the LDOS and the order parameter of a two dimensional lattice of $d$-wave superconductor within the extended Hubbard model, self consistently. The dominant order parameter decays monotonically for [110] interface and $d$-wave order parameter symmetry, while non-monotonically for [100] interface, $d$-wave or $s$-wave order parameter symmetry. The induced extended $s$-wave that decays to zero in the bulk changes sign at the topmost sites at either side of the lattice similarly to the case near impurities, and twin boundaries. The LDOS is symmetric when $\mu=0$ and it becomes asymmetric when $\mu$ deviates from zero. The presence of surface roughness at the corner strongly modifies the quasiparticle properties near the corner. The ZBCP, which is absent for perfect corner consistently to the quasiclassical theory, appears when the roughness at atomic size is introduced due to the oscillatory form of the bound states that decay in the bulk. The last result is beyond the

quasiclassical approximation. The sensitivity of the properties on the atomic scale has to 
be taken into account for the correct interpretation of the experiments on corner junctions and in models that calculate the Josephson current phase relation, or the critical currents in those junctions taking into account the Andreev bound states. 


\section{REFERENCES}

[1] D.J. Scalapino, Phys. Rep. 250, 329 (1995).

[2] D.J. Van Harlingen, Rev. Mod. Phys. 67, 515 (1995).

[3] M. Covington, R. Scheuerer, K. Bloom, and L. Green, Appl. Phys. Lett. 68, 1717 (1996).

[4] J. Lesueur, X. Grison, M. Aprili, and T. Kontos, cond-mat/9909212.

[5] C.R. Hu, Phys. Rev. Lett, 72, 1526 (1994).

[6] Y. Tanuma, Y. Tanaka, M. Yamashiro, and S. Kashiwaya, Phys. Rev . B 57, 7997 (1998).

[7] J.-X. Zhu, B. Friedman, C.S. Ting, Phys. Rev. B 59, 3353 (1999).

[8] N. Stefanakis, J. Phys.: Condens. Matter 13, 1265 (2001).

[9] N. Stefanakis, Phys. Rev. B to appear (2001).

[10] N. Stefanakis, J. Phys.: Condens. Matter 13, 3643 (2001).

[11] P.I. Soininen, C. Kallin, and A. J. Berlinsky, Phys. Rev. B 50, 13883 (1994).

[12] Y. Wang and A.H. MacDonald, Phys. Rev. B 52, 3876 (1995).

[13] D.L. Feder, A. Beardsall, A. J. Berlinsky, and C. Kallin, Phys. Rev. B. 56, 5751 (1997).

[14] M. Franz, C. Kallin, and A. J. Berlinsky, Phys. Rev. B 54, 6897 (1996).

[15] P.G. de Gennes, Superconductivity of Metals and Alloys (Benjamin, New York, 1966).

[16] A.M. Martin and J.F. Annett, cond-mat/9811389. 


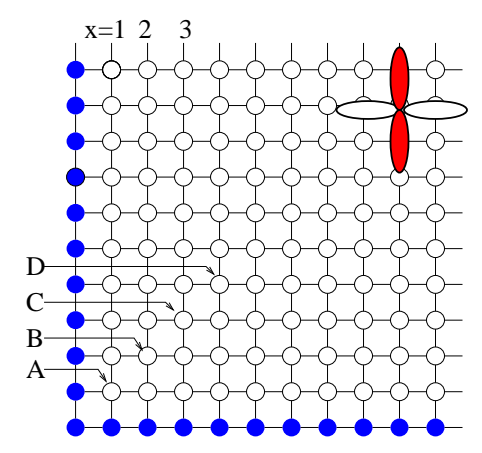

FIG. 1. The square lattice close to the corner. The chemical potential is set to $\mu^{I}=100 t$ at the shaded surface sites.
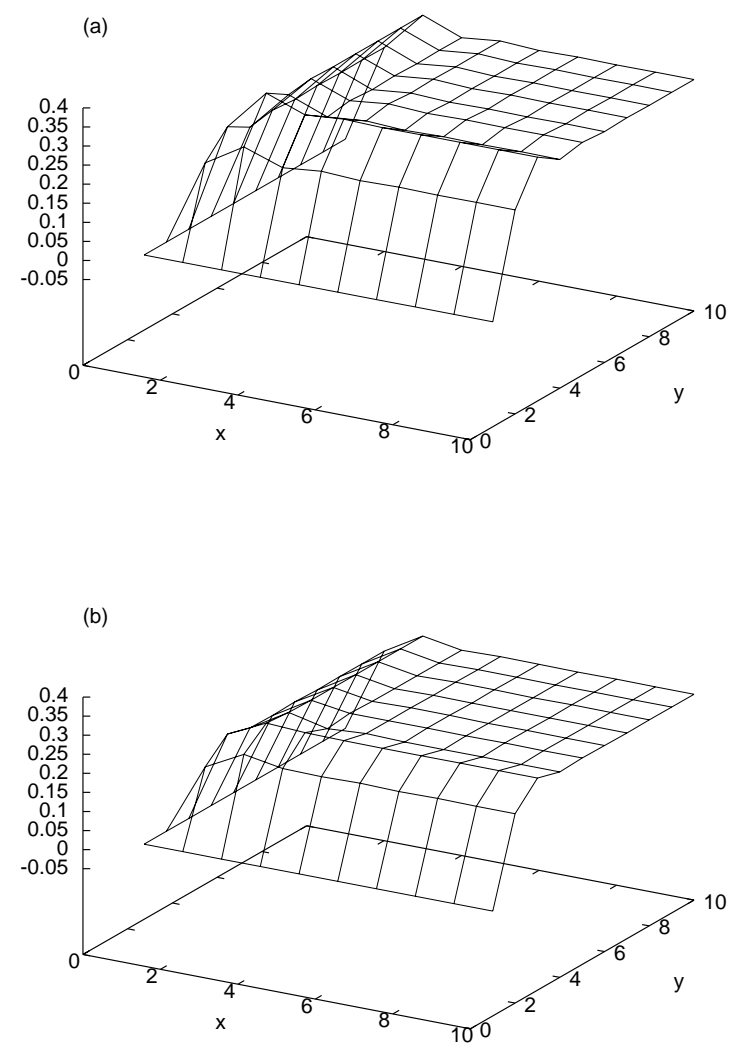

FIG. 2. Spatial dependence of the $d$-wave order parameter close to the corner of a two dimensional square lattice. (a) $\mu=0$, (b) $\mu=t$. 


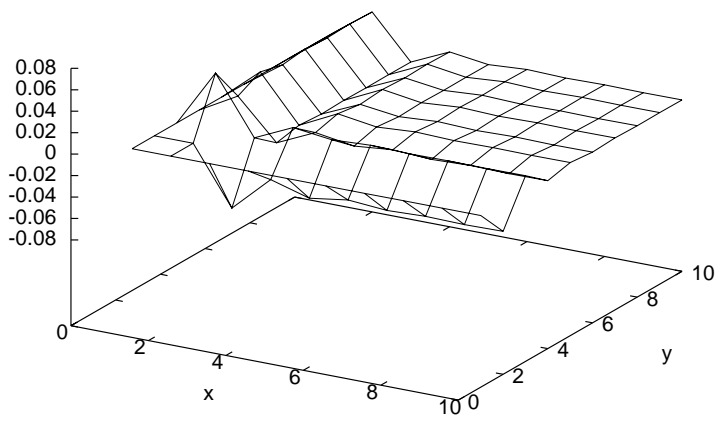

(b)

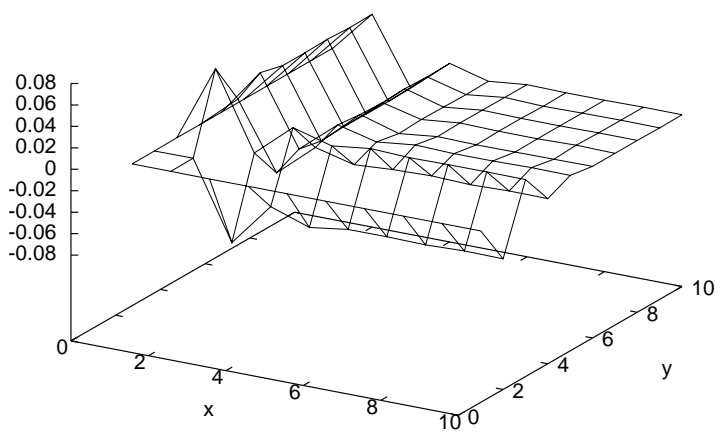

FIG. 3. Spatial dependence of the extended $s$-wave $\Delta_{s}^{e x t}$ order parameter close to the corner of a two dimensional square lattice. (a) $\mu=0$, (b) $\mu=t$. 

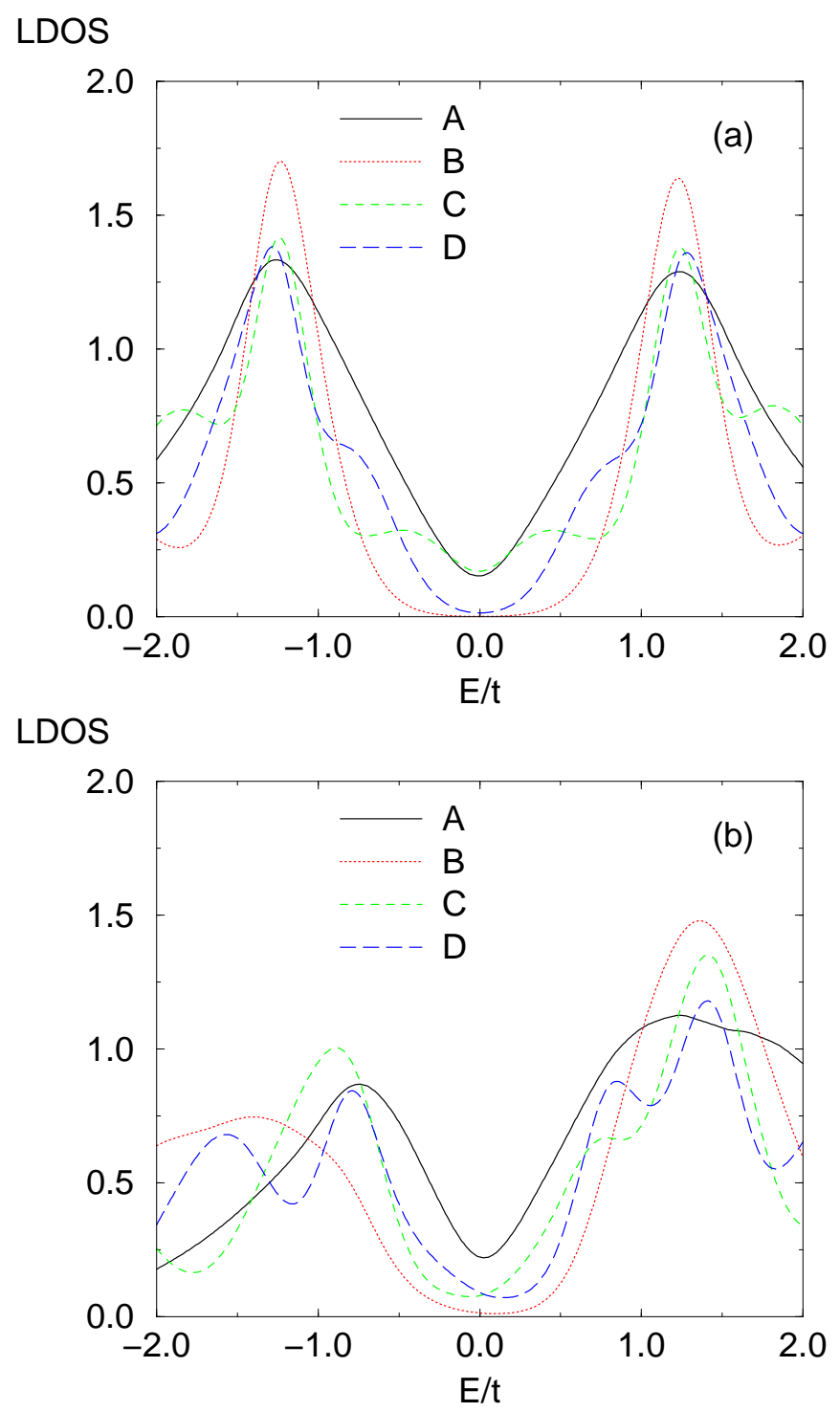

FIG. 4. The local density of states at sites $A, B, C, D$ shown in Fig. 1, along the diagonal of the two dimensional square lattice. (a) $\mu=0$, (b) $\mu=t$. 


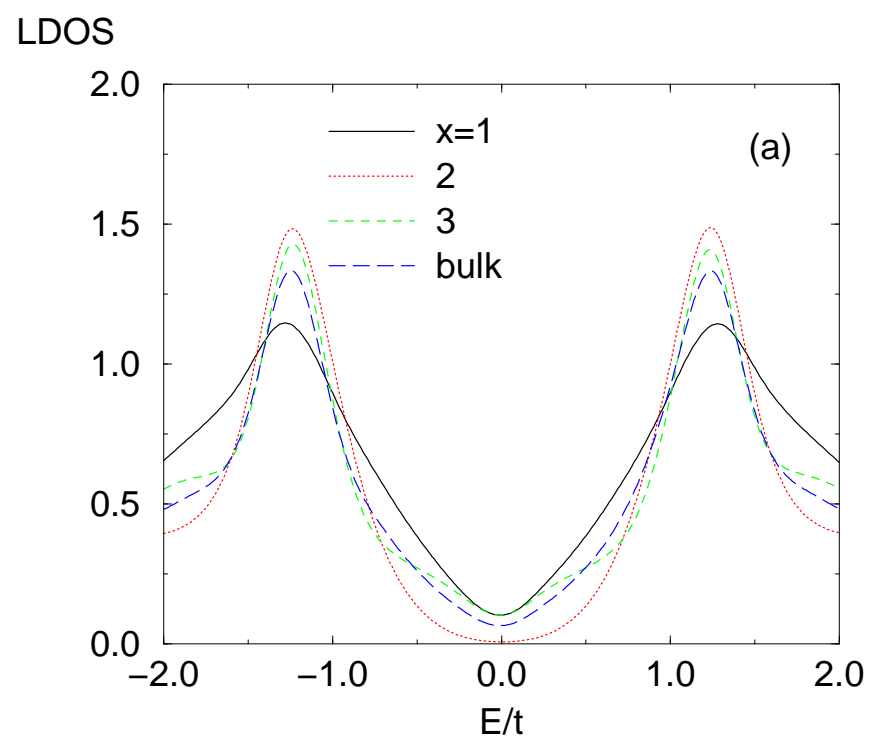

LDOS

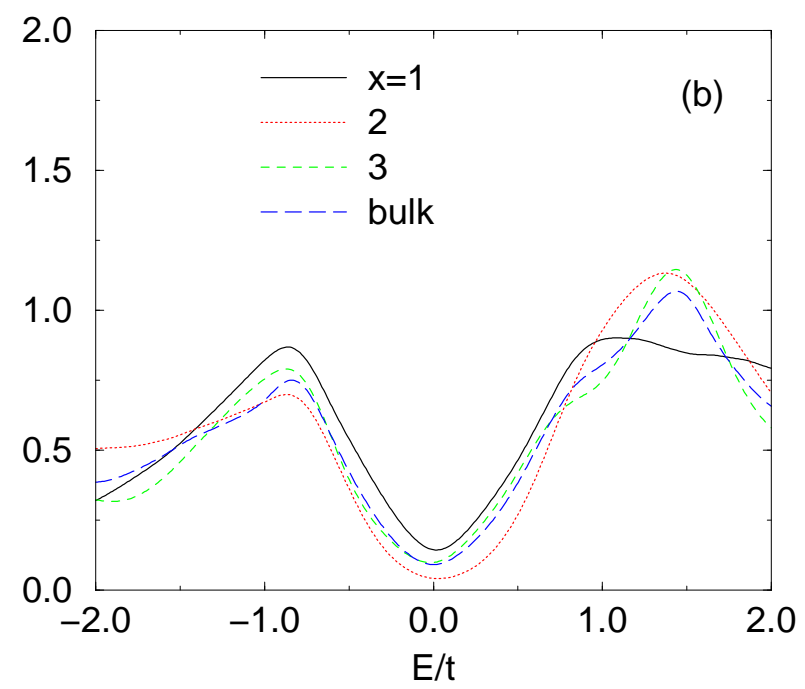

FIG. 5. The local density of states at sites $x=1,2,3$ shown in Fig. 1 along the [100] surface of the two dimensional square lattice, and the bulk density of states. (a) $\mu=0$, (b) $\mu=t$. 


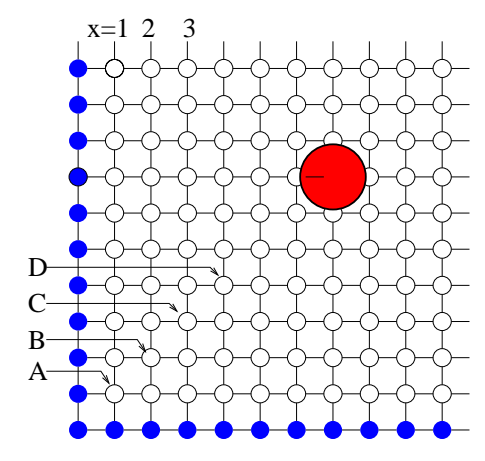

FIG. 6. The square lattice close to the corner. The chemical potential is set to $\mu^{I}=100 t$ at the shaded surface sites.

(a)

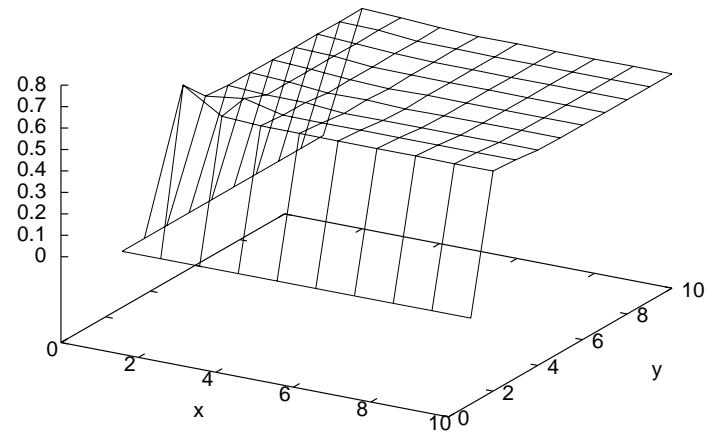

(b)

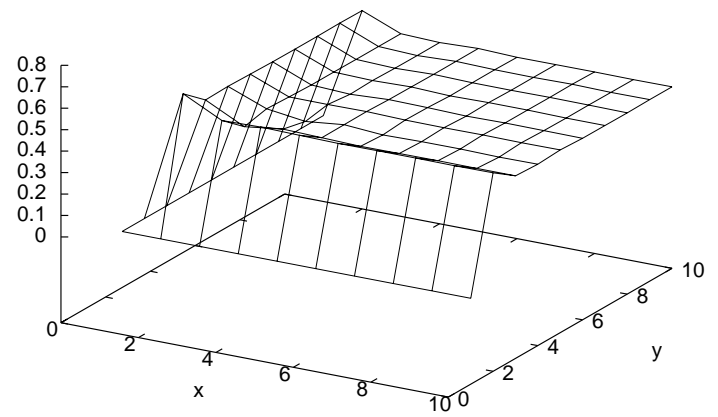

FIG. 7. Spatial dependence of the $s$-wave order parameter close to the corner of a two dimensional square lattice. (a) $\mu=0$, (b) $\mu=t$. 

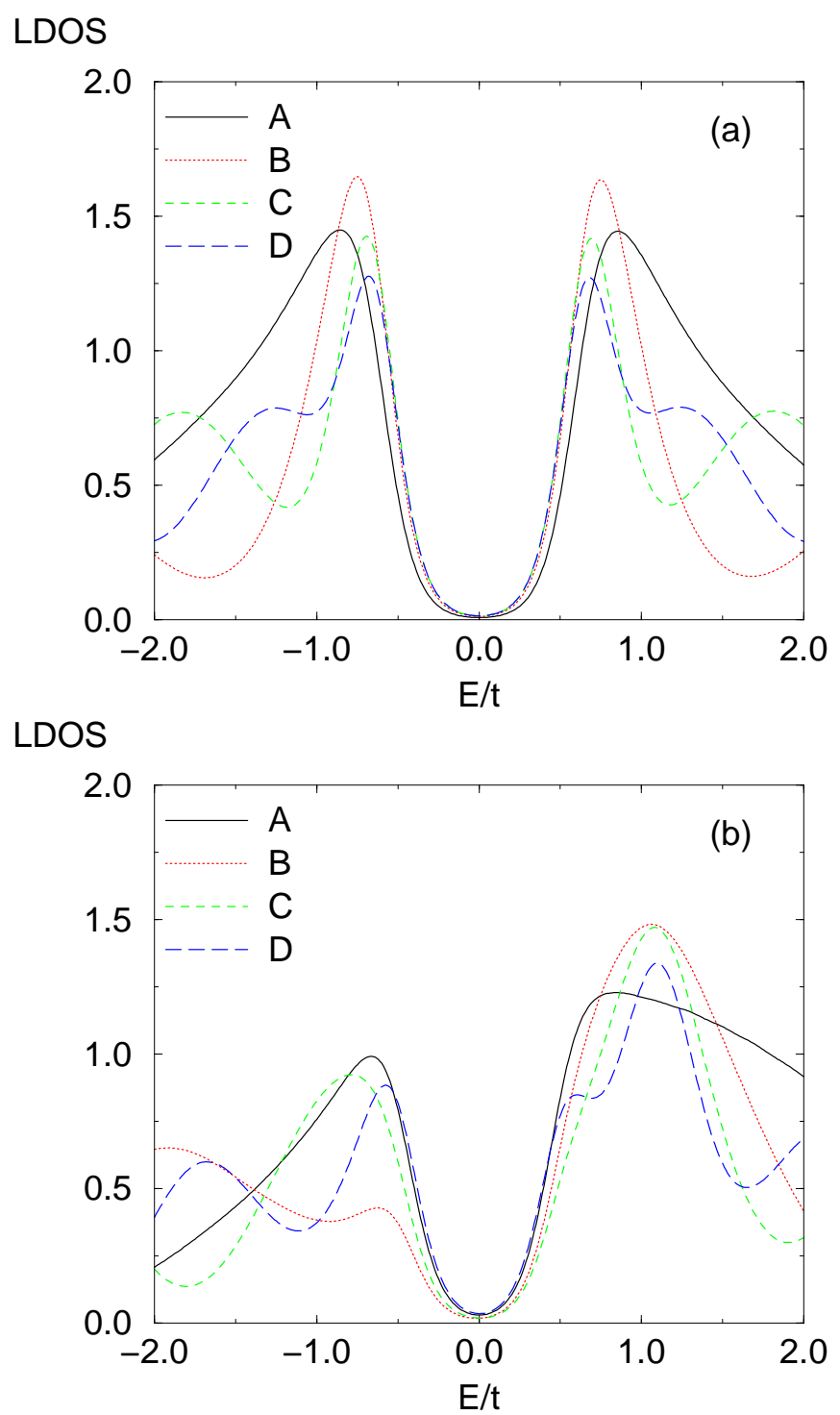

FIG. 8. The local density of states at sites $A, B, C, D$ shown in Fig. 6 along the diagonal of the two dimensional square lattice. The pairing symmetry is $s$. (a) $\mu=0$, (b) $\mu=t$. 


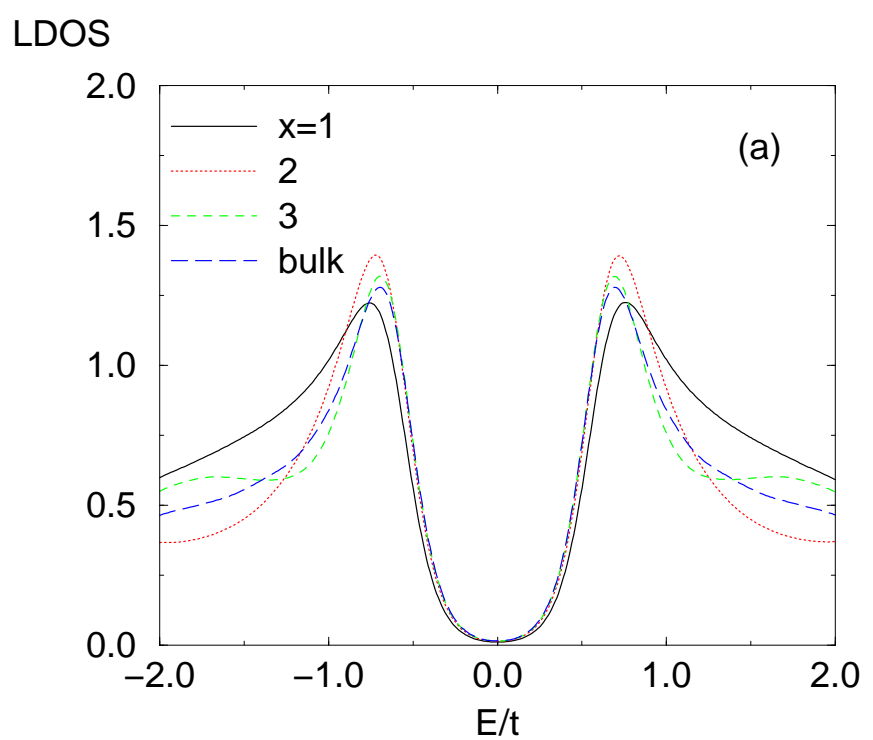

LDOS

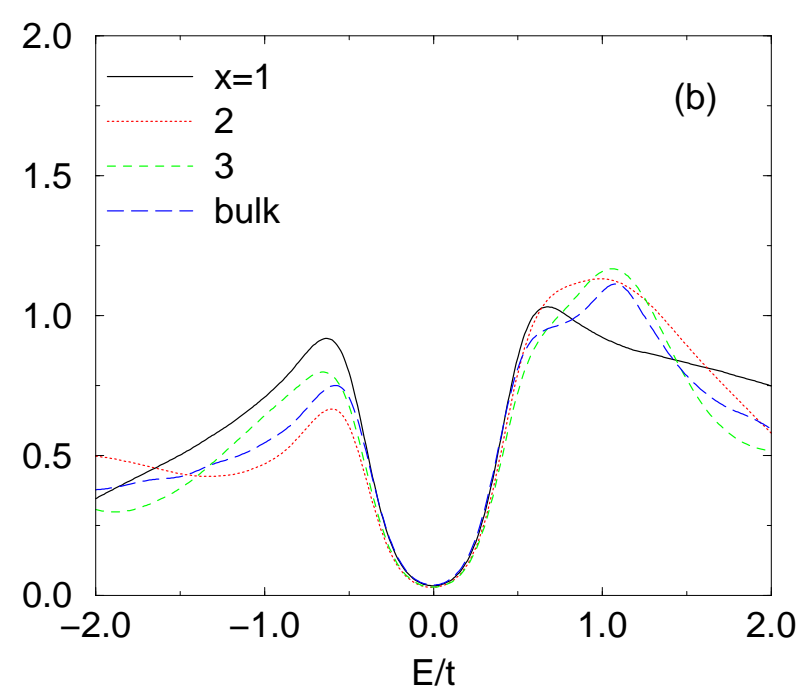

FIG. 9. The local density of states at sites $x=1,2,3$ shown in Fig. 6 along the [100] surface of the two dimensional square lattice, and the bulk density of states. The pairing symmetry is $s$. (a) $\mu=0$, (b) $\mu=t$. 
(a)
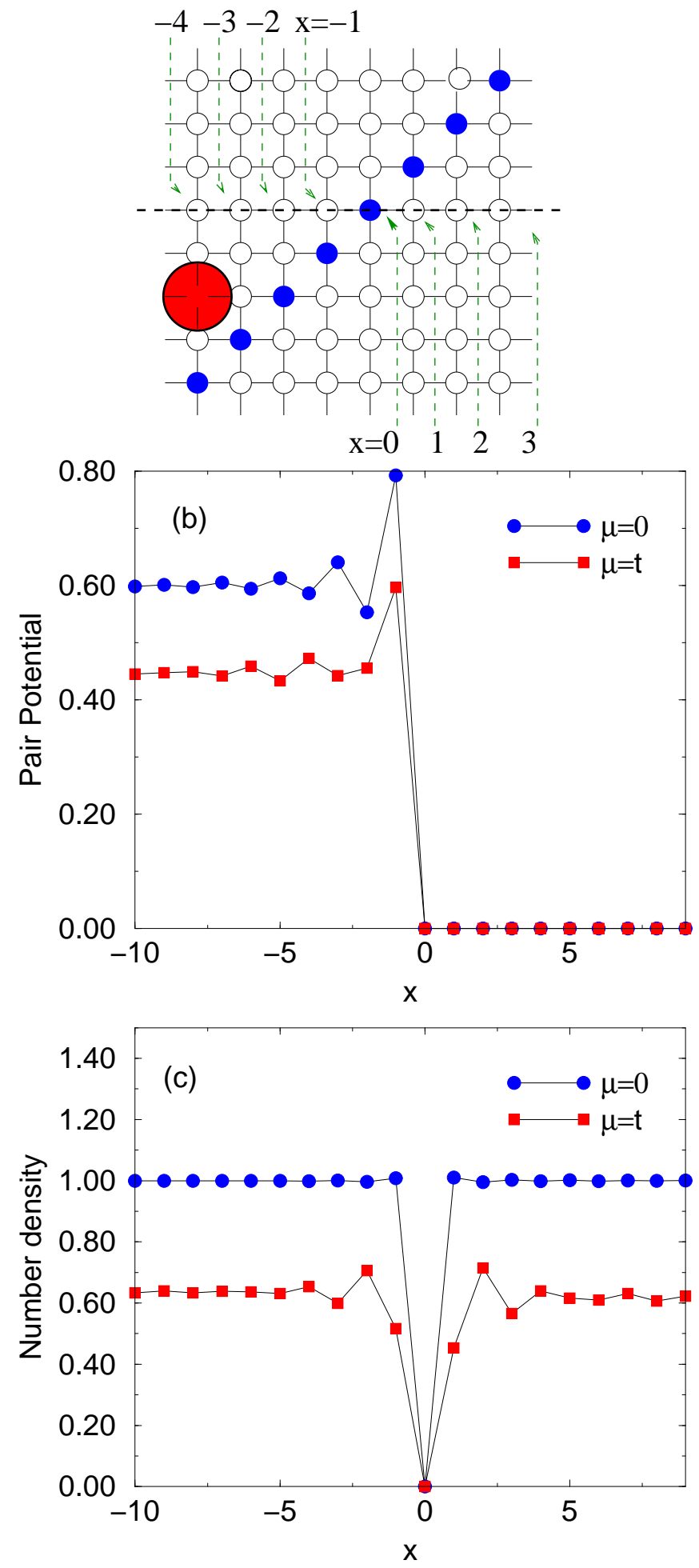

FIG. 10. (a) Spatial distribution of impurities, indicated as solid circles, corresponding to a $s-I-n$ interface along the [110] surface. Also the labeling of the sites along the $x$ direction is shown. (b) The magnitude of the $s$-wave component $\Delta_{s}$ of the superconducting order parameter as a function of $x$, for a $s-I-n$ interface for $\mu$, 1 , The order parameter is calculated along the thick dashed line in direction $x$ shown in (a). (c) The number density $n_{i}$ as a function of $x$ 


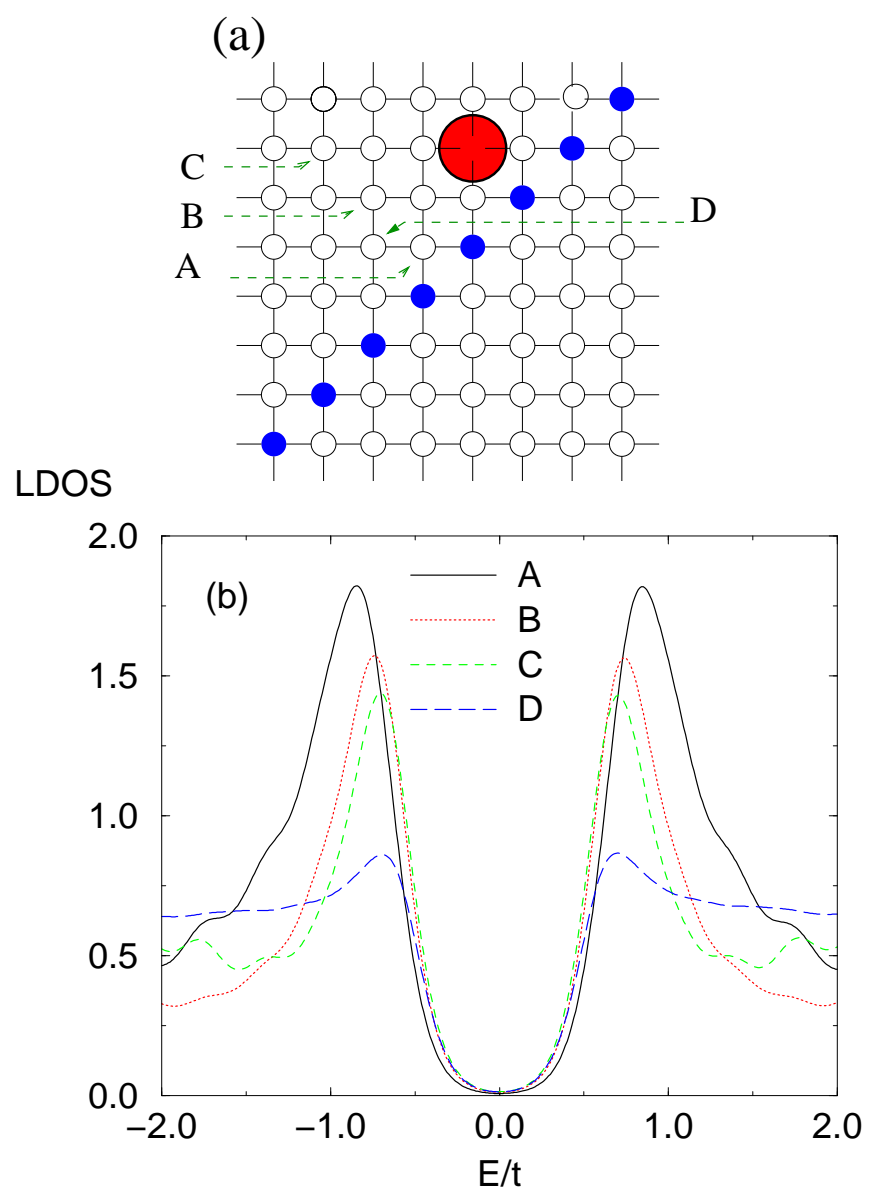

LDOS

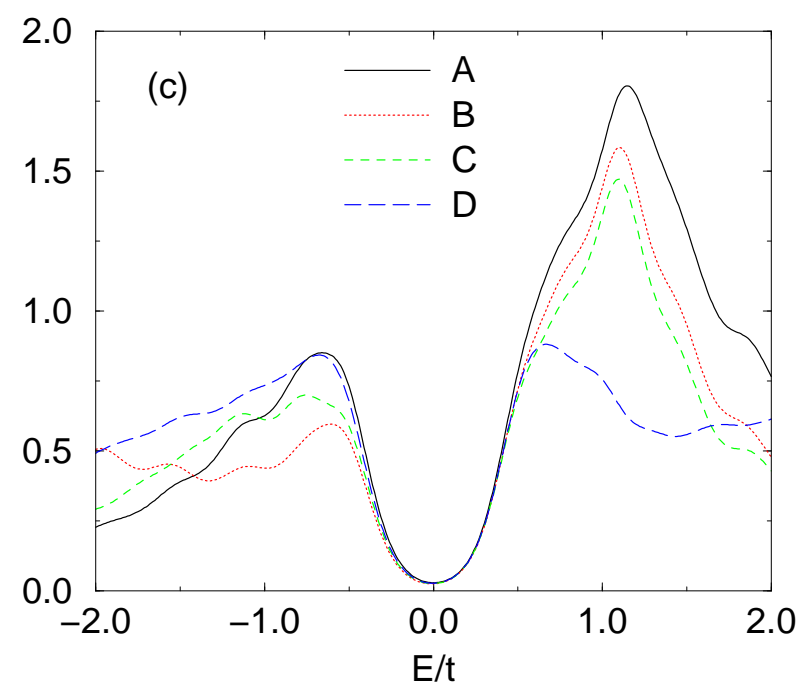

FIG. 11. (a) Spatial distribution of impurities, indicated as solid circles, corresponding to a $s-I-n$ interface along the [110] surface. (b) The local density of states (LDOS) at $A, B, C, D$, shown in (a) of a $s-I-n$ interface for $\mu=0$. (c) The local density of states (LDOS) at $A, B, C, D$, shown in (a) of a $s-I-n$ interface for $\mu=t$. 
(a)
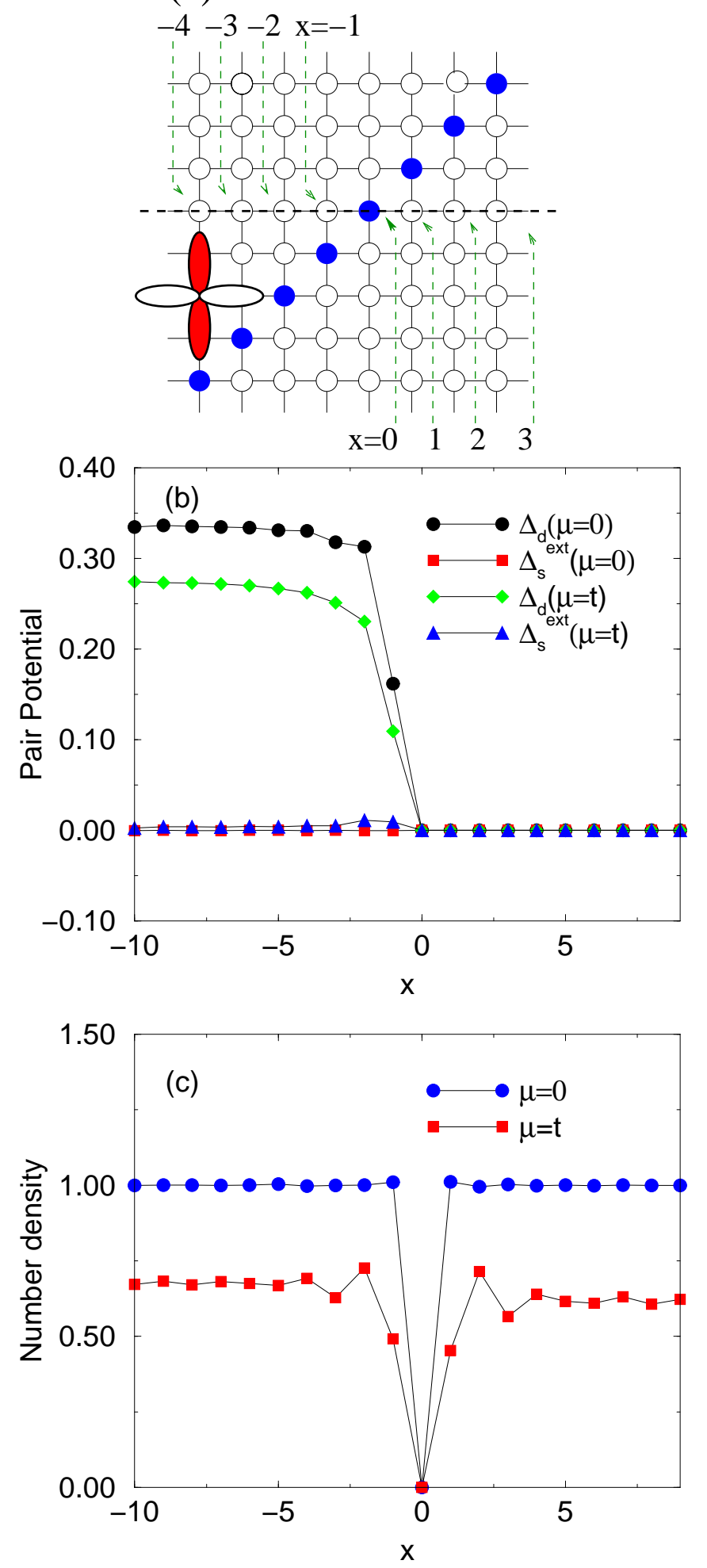

FIG. 12. (a) Spatial distribution of impurities, indicated as solid circles, corresponding to a $d-I-n$ interface along the [110] surface. Also the labeling of the sites along the $x$ direction is shown. (b) The magnitude of the $d$-wave component $\Delta_{d}$ and the extended $s$-wave component 23 $\left(\Delta_{s}^{e x t}\right)$ of the superconducting order parameter as a function of $x$, for a $d-I-n$ interface for 


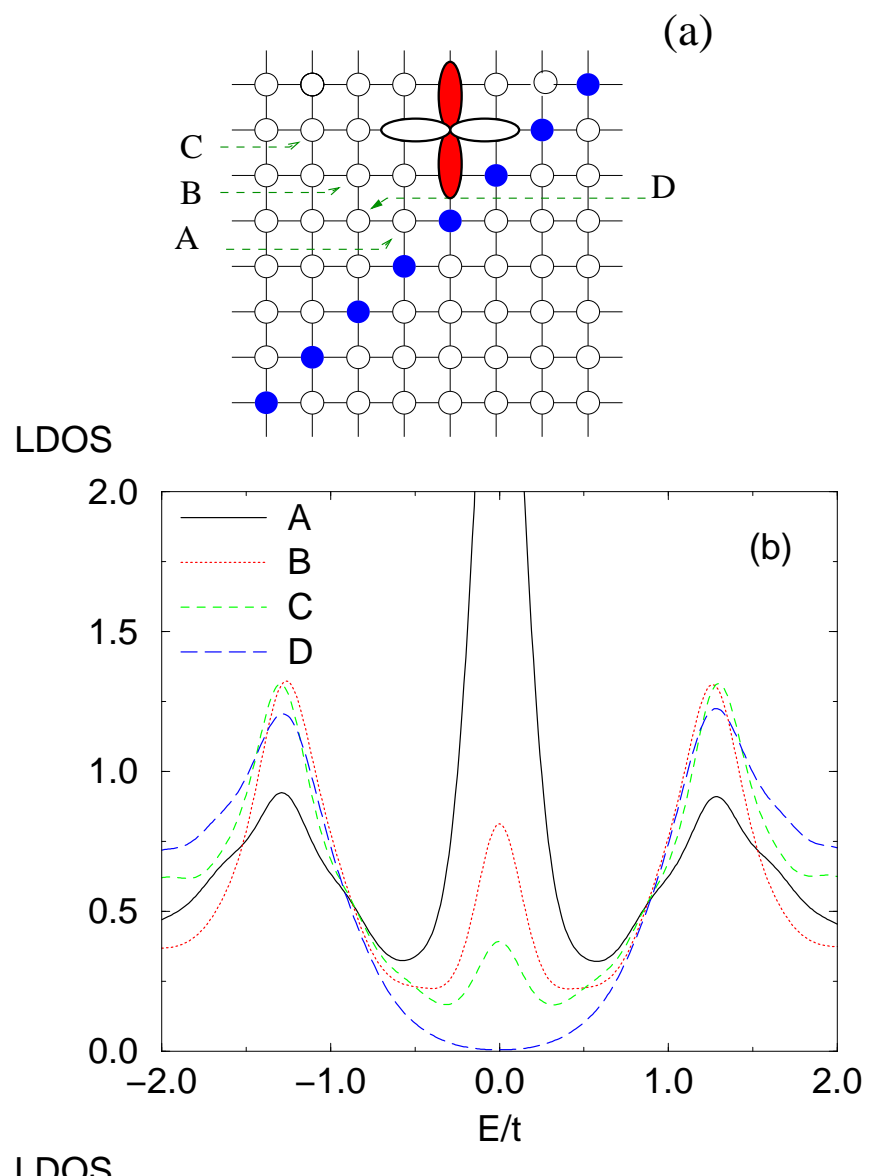

LDOS

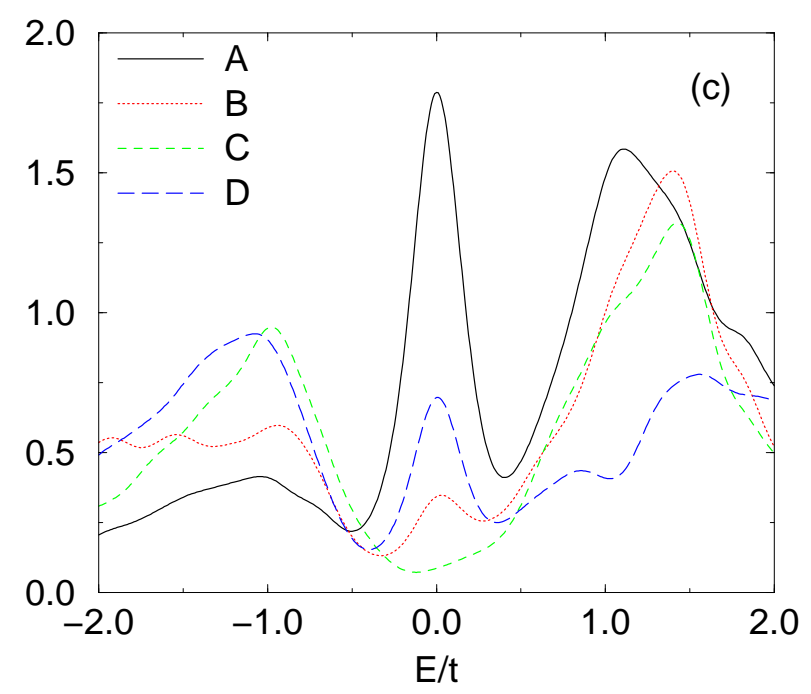

FIG. 13. (a) Spatial distribution of impurities, indicated as solid circles, corresponding to a $d-I-n$ interface along the [110] surface. (b) The local density of states (LDOS) at $A, B, C, D$, shown in (a) of a $d-I-n$ interface for $\mu=0$. (c) The local density of states (LDOS) at $A, B, C, D$, shown in (a) of a $d-I-n$ interface for $\mu=t$. 
(a)

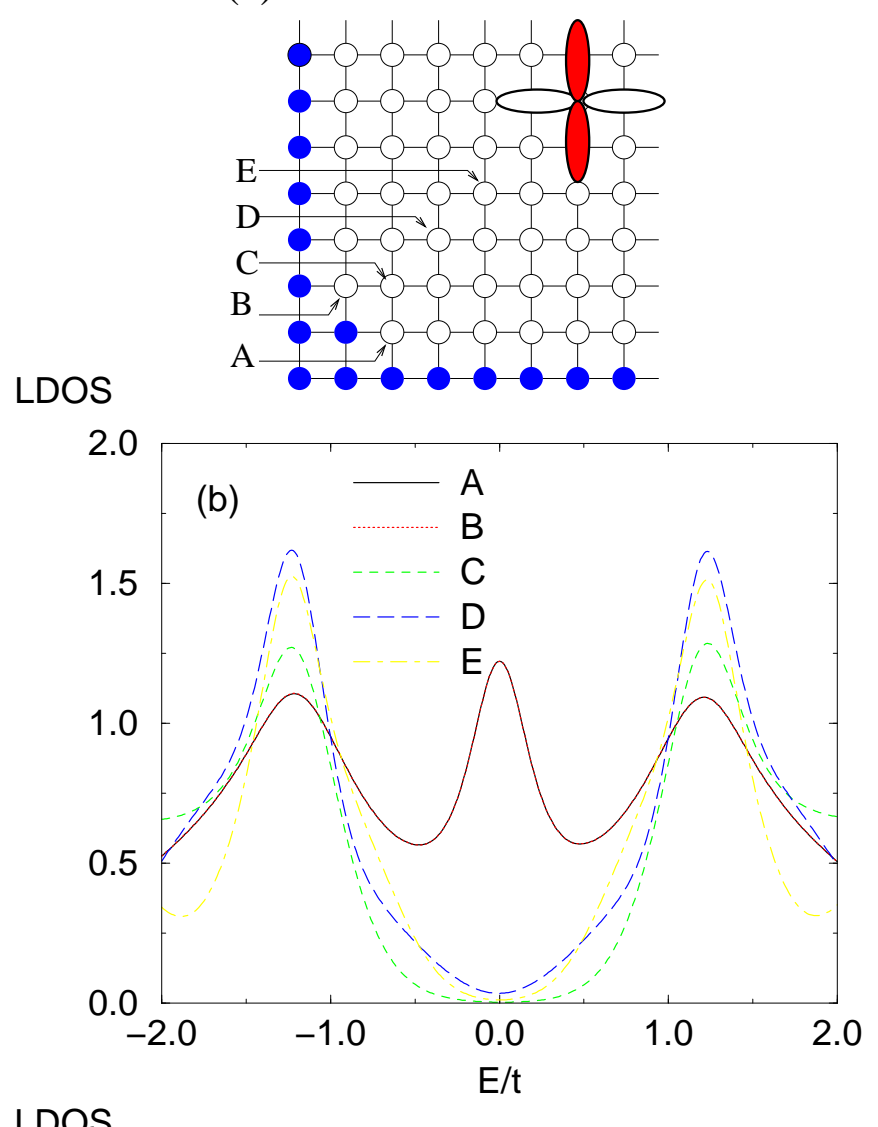

LDOS

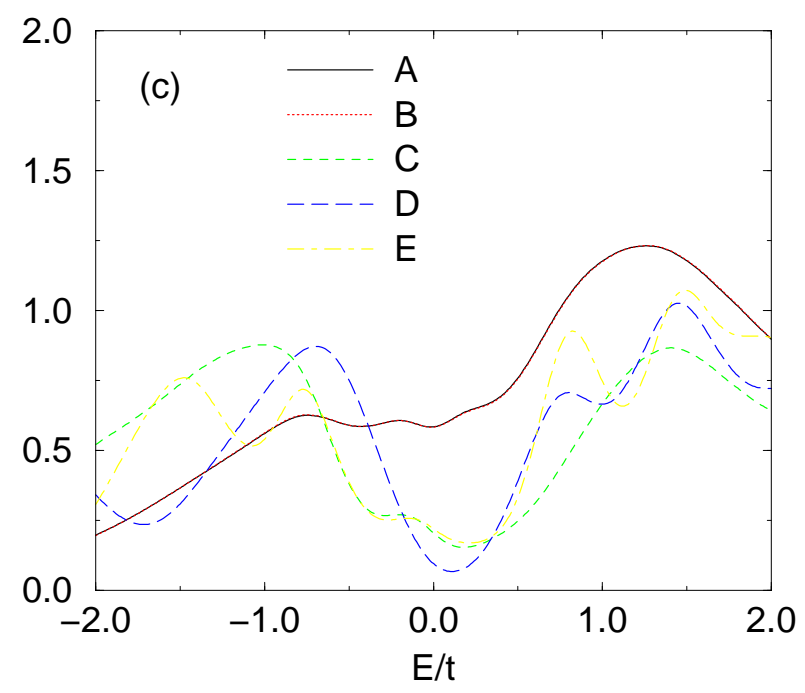

FIG. 14. (a) Spatial distribution of impurities corresponding to the corner surface, with a $1 \times 1$ step structure. The chemical potential is set to $\mu^{I}=100 t$ at the shaded surface sites. (b) The local density of states at the specified sites $A, B, C, D, E$ shown in (a) for $\mu=0$. (c) The local density of states at the specified sites $A, B, C, D, E$ shown in (a) for $\mu=t$. 


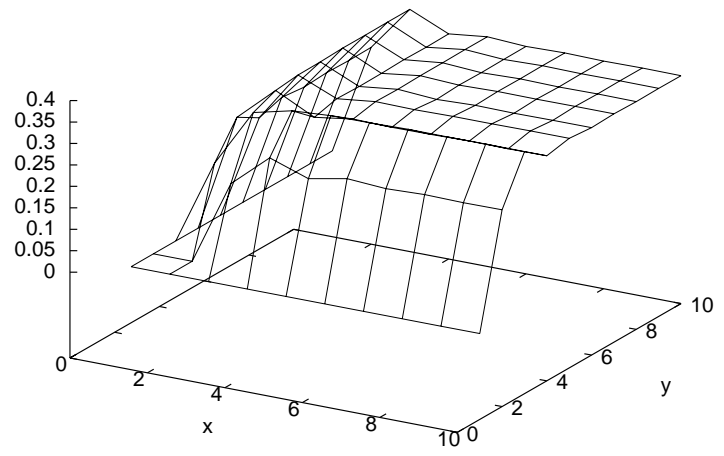

(b)

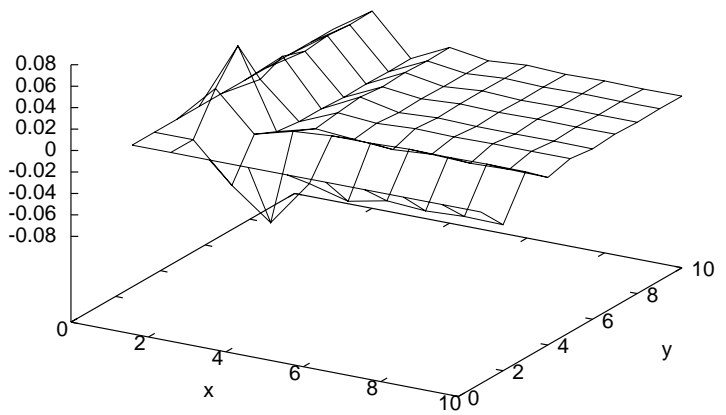

FIG. 15. Spatial dependence of the (a) $d$-wave and (b) the extended $s$-wave order parameter for the specified geometry of Fig. 14(a). 


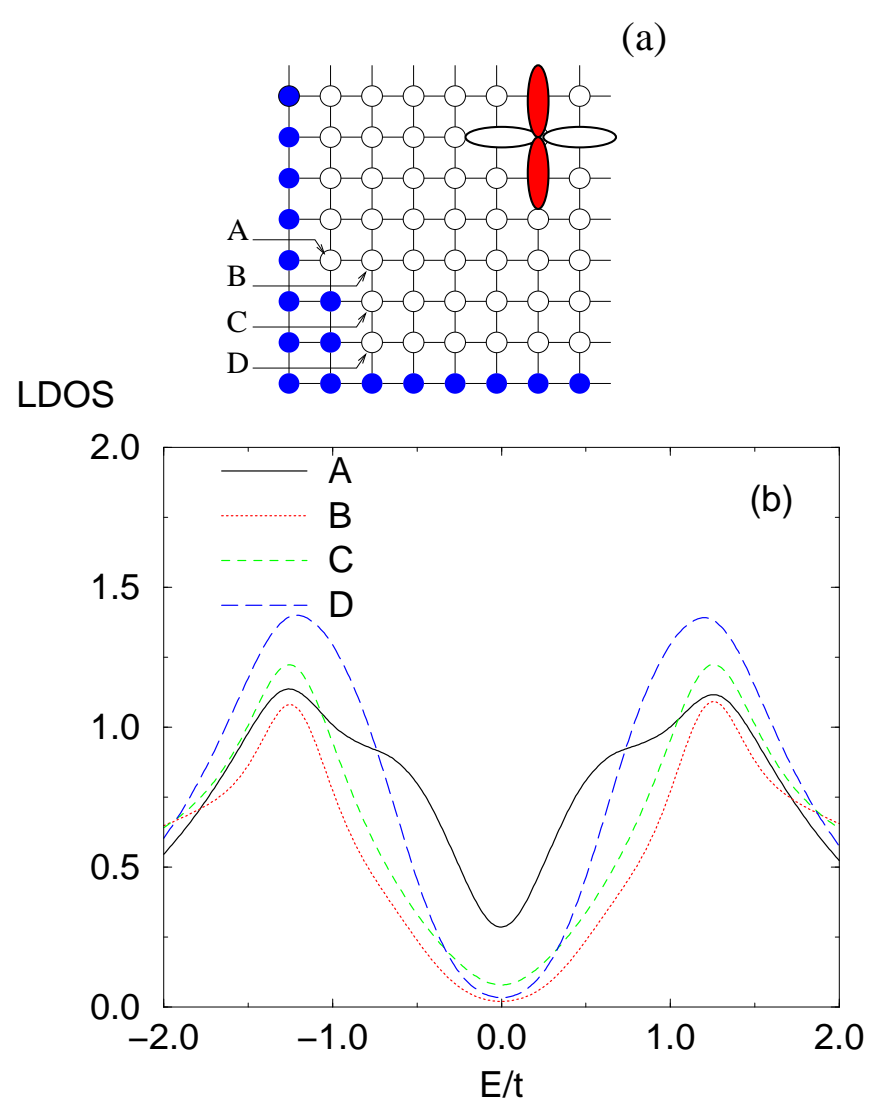

LDOS

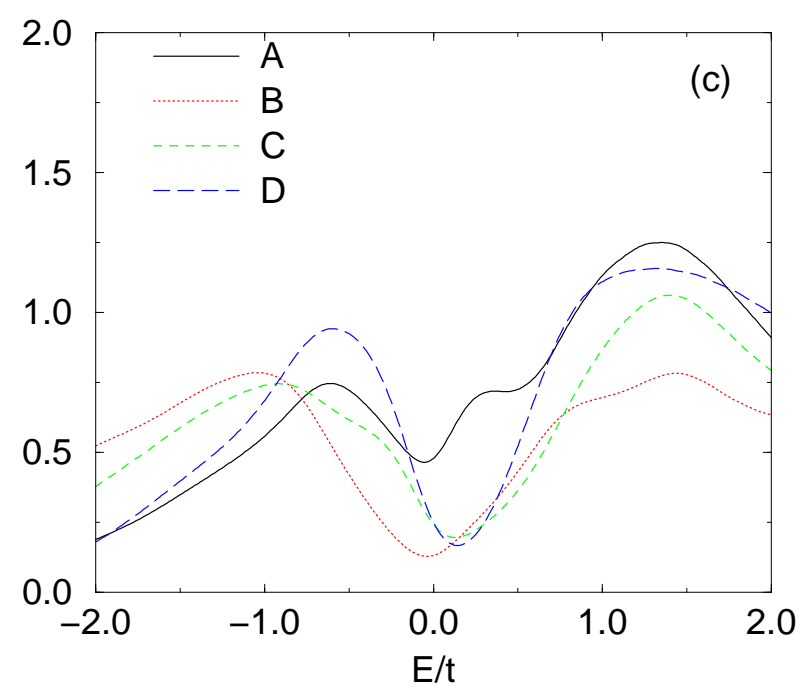

FIG. 16. (a) Spatial distribution of impurities corresponding to the corner surface, with a $1 \times 2$ step structure. The chemical potential is set to $\mu^{I}=100 t$ at the shaded surface sites. (b) The local density of states at the specified sites $A, B, C, D$ shown in (a) for $\mu=0$. (c) The local density of states at the specified sites $A, B, C, D$ shown in (a) for $\mu=t$. 


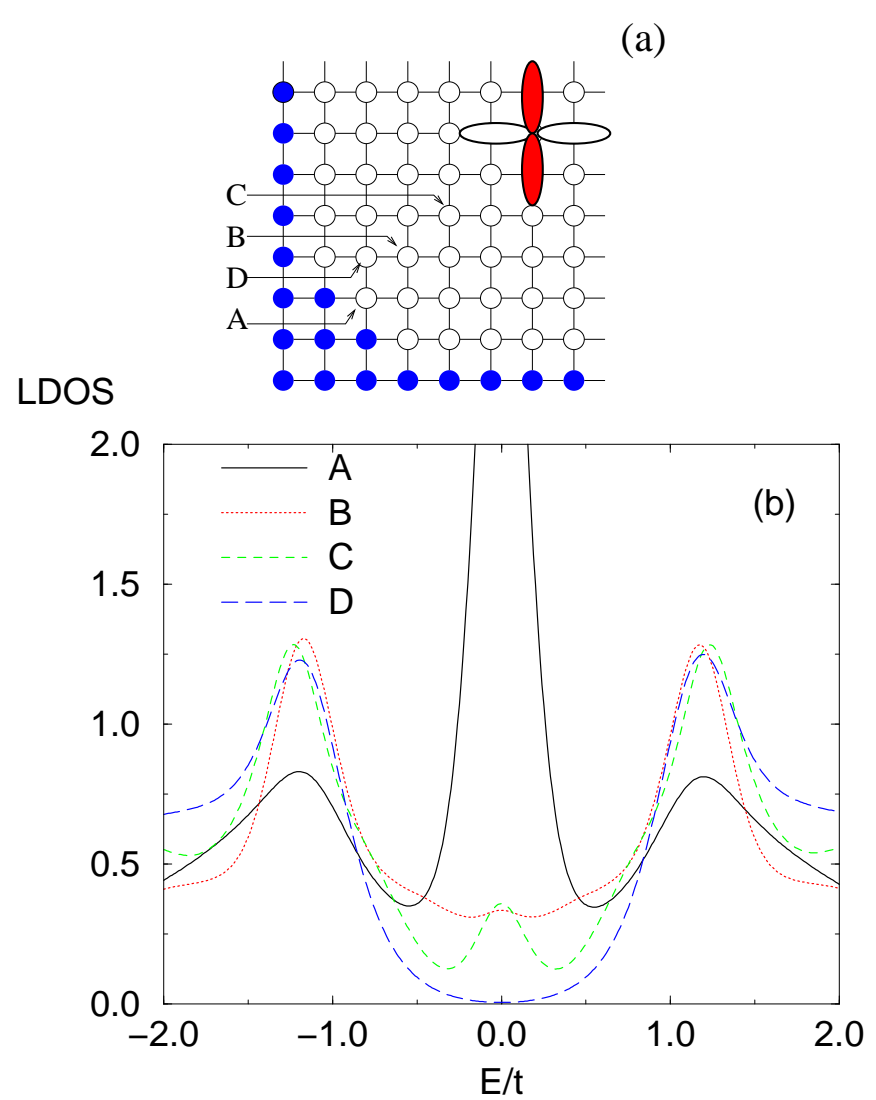

LDOS

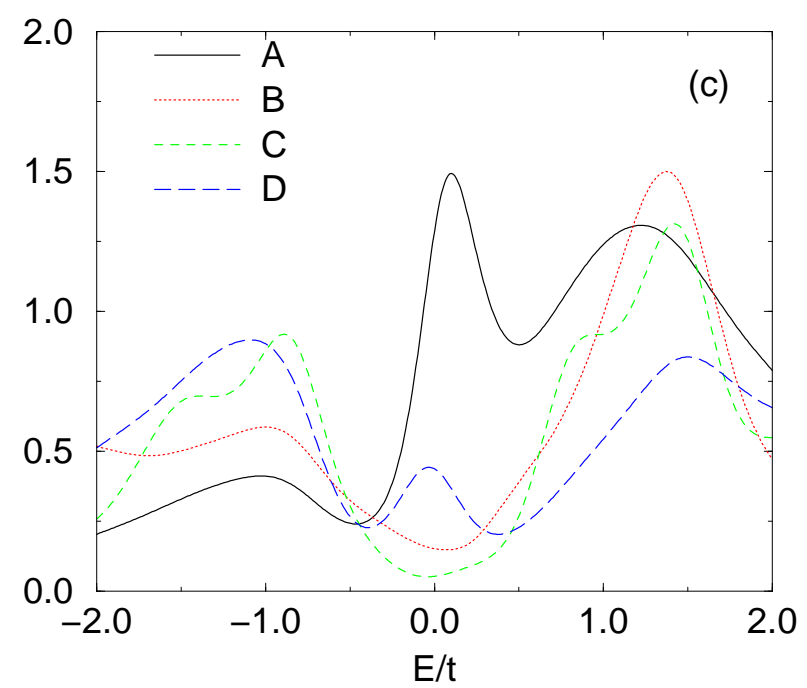

FIG. 17. (a) Spatial distribution of impurities corresponding to the corner surface, with a [110] structure. The chemical potential is set to $\mu^{I}=100 t$ at the shaded surface sites. (b) The local density of states at the specified sites $A, B, C, D$ shown in (a) for $\mu=0$. (c) The local density of states at the specified sites $A, B, C, D$ shown in (a) for $\mu=t$. 